\title{
WEAK-STAR CLOSED ALGEBRAS AND GENERALIZED BERGMAN KERNELS
}

\author{
KARIM SEDDIGHI
}

\begin{abstract}
In this paper we will characterize the weak-star closed algebra $\mathbb{Q}$ generated by the canonical model associated with a generalized Bergman kernel defined on a domain $G$ in the plane, whose spectrum is a spectral set. In fact, $Q$ equals the space $H^{\infty}\left(G_{0}\right)$ of all bounded analytic functions on an appropriate set $G_{0}$ containing $G$.
\end{abstract}

1. Introduction. A compact subset $K$ of the plane is a spectral set for $T \in \mathcal{L}(\mathcal{H})$ if it contains the spectrum of $T, \sigma(T)(=\{\lambda \in \mathbf{C}: T-\lambda$ is not invertible $\})$ and $\|f(T)\| \leqslant \max \{|f(z)|: z \in K\}$ for all rational functions $f$ with poles off $K$. An operator $T$ whose spectrum is a spectral set for $T$ is called a von Neumann operator.

Let $T$ be the canonical model associated with a generalized Bergman kernel defined on an open connected subset $\Omega$ of the plane such that $\sigma(T)=\bar{\Omega}$ is a spectral set for $T$. We will show the existence of a simply connected open set $\Omega_{0}$ containing $\Omega$ such that the smallest weak * closed subalgebra of $\mathcal{L}(\mathcal{H})$ containing $T$ and the identity is actually isometrically isomorphic to the space $H^{\infty}\left(\Omega_{0}\right)$ of all bounded analytic functions in $\Omega_{0}$.

2. Preliminaries. For completeness purposes we include some preparatory material of a general nature. We will denote the Banach space of trace-class operators in $\mathcal{L}(\mathcal{H})$ with the trace norm \|\|$_{1}$ by $\bigodot_{1}$. Recall from [4, Theorem 8, p. 105] that setting $\langle T, A\rangle=\operatorname{tr}(A T), A \in \mathcal{L}(\mathcal{H}), T \in \mathcal{C}_{1}$, defines a bilinear functional on $\mathcal{C}_{1} \times \mathcal{E}(\mathcal{H})$ that allows us to identify $\bigodot_{1}^{*}$ with $\mathcal{E}(\mathcal{H})$. We refer to the weak * topology $\mathcal{L}(\mathcal{H})$ inherits as a dual as the $w^{*}$-topology. Some authors choose to call this topology "ultra weak", though it is stronger than what is commonly referred to as the "weak operator topology". We denote the weak operator topology by WOT.

For an operator $T$ in $\mathcal{L}(\mathcal{H})$, let $\mathcal{Q}(T)$ denote the smallest weak * closed subalgebra of $\mathcal{E}(\mathcal{H})$ containing $T$ and 1 , and we call $Q(T)$ the weak * closed algebra generated by $T$. (It is clear that $Q(T)$ is the closure in the weak * topology of the algebra of all polynomials $p(T)$ in $T$.)

Let $K$ be a compact subset of the complex plane C, and let $R(K)$ denote the algebra of all continuous complex-valued functions on $K$ which can be approximated

Received by the editors October 5, 1982 and, in revised form, June 7, 1983; presented to the Society, January 5, 1983.

1980 Mathematics Subject Classification. Primary 47B20; Secondary 47A25.

Key words and phrases. Spectral set, weak-star closed algebra, generalized Bergman kernel. 
uniformly on $K$ by rational functions whose poles all lie outside $K . R(K)$ is a Dirichlet algebra on $\partial K$ if $\left.\operatorname{Re} R(K)\right|_{\partial K}$ is dense in $C_{\mathbf{R}}(\partial K)$. That is, the real parts of the functions in $R(K)$ when restricted to $\partial K$ are dense in the continuous real-valued functions on $\partial K$.

If $R(K)$ is Dirichlet and $z$ is in the interior of $K$ (denoted by $K^{\circ}$ ), then there exists a unique measure $m_{z}$ supported on $\partial K$ such that $\int f d m_{z}=f(z)$ for all $f \in R(K)$. Let $\left\{G_{n}\right\}_{n=1}^{\infty}$ be the components of $K^{\circ}$ and fix $z_{n} \in G_{n}$ for all $n \geqslant 1$. Set $m=\sum_{n=1}^{\infty} 2^{-n} m_{z_{n}}$. This $m$ will be referred to as the harmonic measure on $\partial K$. We let $H^{\infty}(\partial K)$ denote the weak * closure of $R(K)$ in $L^{\infty}(m)$. The weak * topology is denoted by w*. It is a well-known fact that the definitions of $L^{\infty}(m)$ and $H^{\infty}(\partial K)$ are independent of the sequence $\left\{z_{n}\right\}$ used to define the measure $m$.

For each function $f$ in $H^{\infty}(\partial K)$ we define a function $\hat{f}$ on $K^{\circ}$ by $\hat{f}(z)=\int f d m_{z}$. It is a standard result that $\hat{f}$ is a bounded analytic function in $K^{\circ}[5$, Lemma 4.2, p. 5]. It is also shown there that whenever $R(K)$ is a Dirichlet algebra, the map $f \rightarrow \hat{f}$ is an isometric isomorphism of $H^{\infty}(\partial K)$ onto $H^{\infty}\left(K^{\circ}\right)$, the space of bounded analytic functions in $K^{\circ}$. It is customary not to distinguish between the two spaces $H^{\infty}(\partial K)$ and $H^{\infty}\left(K^{0}\right)$, and we will also follow this custom. We will also use, either explicitly or implicitly, another well-known fact from function theory which states that whenever $R(K)$ is a Dirichlet algebra, then the components of $K^{\circ}$ are simply connected.

For $\Omega$ an open connected (not necessarily simply connected) subset of the plane and $\alpha$ an ordinal number, the set $\Omega_{\alpha}$ can be defined as in Sarason [6, p. 525]. For the benefit of the reader we will reiterate the necessary definitions.

Recall that a connected open subset of the plane is called a domain. If $B$ is a bounded domain in the plane, then the Carathéodory hull (or C-hull) of $B$ is the complement of the closure of the unbounded component of the complement of the closure of $B$. We denote the $\mathcal{C}$-hull of $B$ by $B^{*}$. Loosely speaking, $B^{*}$ can be described as the interior of the outer boundary of $B$, and in analytic terms it can be defined as the interior of the set of all points $z_{0}$ in the plane such that $\left|p\left(z_{0}\right)\right| \leqslant$ $\sup \{|p(z)|: z \in B\}$ for all polynomials $p$. The components of $B^{*}$ are simply connected; in fact, it is a simple matter to show that each of these components has a connected complement. We denote by $B_{1}$ the component of $B^{*}$ that contains $B$.

Again let $B$ be a bounded domain in the plane. For any simply connected domain $E$ containing $B$ we can define the relative hull of $B$ in $E$, or the $E$-hull of $B$, to be the interior of the set of all points $z_{0}$ in $E$ such that $\left|f\left(z_{0}\right)\right| \leqslant \sup \{|f(z)|: z \in B\}$ for every function $f$ bounded and analytic in $E$.

Now let $\Omega$ be a bounded domain in the plane. We have already defined $\Omega_{1}$ to be the component of the $\mathcal{C}$-hull of $\Omega$ that contains $\Omega$. We now define inductively for every countable ordinal number $\alpha$ a simply connected domain $\Omega_{\alpha}$ containing $\Omega$ as follows. If $\alpha$ has an immediate predecessor we let $\Omega_{\alpha}$ be the component of the $\Omega_{\alpha-1}$-hull of $\Omega$ that contains $\Omega$. If $\alpha$ has no immediate predecessor we define $\Omega_{\alpha}$ to be the component of the interior of $\cap_{\beta<\alpha} \Omega_{\beta}$ that contains $\Omega$. (It is easily verified that $\Omega_{\alpha}$ then has a connected complement, and so is simply connected.) It is shown in [6, p. 525] that there is a least countable ordinal $\gamma$ such that $\Omega_{\gamma}=\Omega_{\gamma+1}$. We call $\gamma$ the order of $\Omega$. Obviously $\Omega_{\alpha}=\Omega_{\gamma}$ for all $\alpha \geqslant \gamma$. For convenience we set $\Omega_{0}=\Omega_{\gamma}$. 
It is easy to show that for every $\alpha, R\left(\bar{\Omega}_{\alpha}\right)$ is a Dirichlet algebra. Therefore it makes sense to talk about $H^{\infty}\left(\partial \bar{\Omega}_{\alpha}\right)$, the $\mathrm{w}^{*}$-closure of $R\left(\bar{\Omega}_{\alpha}\right)$ in $L^{\infty}(m)$, where $m$ is the harmonic measure on $\partial \bar{\Omega}_{\alpha}$. We should also point out that $\partial \Omega_{\alpha}=\partial \bar{\Omega}_{\alpha}$.

The following presentation on the generalized Bergman kernels is taken from [3] and will be needed in the sequel.

For $1 \leqslant n \leqslant \infty$ let $l_{n}^{2}=\left\{\xi=\left\{a_{k}\right\}_{k=1}^{n}: a_{k} \in \mathbf{C}\right.$ and $\left.\sum_{k=1}^{n}\left|a_{k}\right|^{2}<\infty\right\}$. Given a set $\Lambda$, a functional Hilbert space on $\Lambda$ will be any Hilbert space which is a linear subspace of $\mathscr{F}\left(\Lambda, l_{n}^{2}\right)$, the linear space of all $l_{n}^{2}$-valued functions on $\Lambda$, for some $1 \leqslant n \leqslant \infty$.

By a kernel function on $\Lambda$ we mean a function $K: \Lambda \times \Lambda \rightarrow \mathscr{L}\left(l_{n}^{2}\right)$ satisfying:

(a) $K(\lambda, \mu)^{*}=K(\mu, \lambda)($ all $\lambda, \mu \in \Lambda)$;

(b) for every integer $k, k \geqslant 1$, and every collection $\left\{\lambda_{1}, \ldots, \lambda_{k}\right\} \subset \Lambda$, the $k \times k$ operator matrix $K_{k}=\left(K\left(\lambda_{i}, \lambda_{j}\right)\right)_{1 \leqslant i, j \leqslant k}$ is a positive operator on $l_{n}^{2} \otimes \mathbf{C}^{k}$.

If, in addition, the $k \times k$ operator matrix $K_{k}$ in (b) is injective for every collection $\left\{\lambda_{1}, \ldots, \lambda_{k}\right\} \subset \Lambda$, all $k \geqslant 1$, we say $K$ is strictly positive.

Let $\mathscr{K}$ be a functional Hilbert space on $\Lambda$. If for every $\lambda \in \Lambda$ and $\xi \in l_{n}^{2}$, $K(\lambda, \cdot) \xi \in \mathscr{K}$ and $\langle f, K(\lambda, \cdot) \xi\rangle_{\mathscr{K}}=\langle f(\lambda), \xi\rangle$, for every $f \in \mathcal{K}$, then we say $\mathscr{K}$ has a reproducing kernel on $\Lambda$, and $K$ will be called the reproducing kernel for $\mathscr{K}$. It is well known that $K$ is unique.

Note that if $K$ is a strictly positive kernel function on $\Lambda$, it gives rise to a functional Hilbert space on $\Lambda$ with reproducing kernel $K$. To see this, let $\mathscr{D}(\Lambda)$ be the linear subspace of $\mathscr{F}\left(\Lambda, l_{n}^{2}\right)$ consisting of all functions of the form $f=$ $\sum_{i=1}^{k} K\left(\lambda_{i}, \cdot\right) \xi_{i}$, for $\lambda_{i} \in \Lambda, \xi_{i} \in l_{n}^{2}(i=1, \ldots ., k), k \geqslant 1$. If $g$ is another function in D $(\Lambda), g=\sum_{j=1}^{k} K\left(\lambda_{j}, \cdot\right) \eta_{j}$, let $\langle f, g\rangle_{K}=\sum_{i, j=1}^{k}\left\langle K\left(\lambda_{i}, \lambda_{j}\right) \xi_{i}, \eta_{j}\right\rangle$. It readily follows that $\langle\cdot, \cdot\rangle_{K}$ is an inner product on $\mathscr{D}(\Lambda)$. It is easy to show that a Cauchy sequence in $\mathscr{D}(\Lambda)$ is pointwise convergent, and hence the completion $\mathcal{K}_{K}$ of $\mathscr{D}(\Lambda)$ with respect to $\langle\cdot, \cdot\rangle_{K}$ is a linear subspace of $\mathscr{F}\left(\Lambda, l_{n}^{2}\right)$. Furthermore, by construction of $\mathcal{K}_{K}$, it follows that $K$ is the reproducing kernel for $\mathcal{K}_{K}$. We also observe that if $K$ is a strictly positive kernel function which is already a reproducing kernel for a functional Hilbert space $\mathscr{K}$ on $\Lambda$, then $\mathcal{K}_{K}=\mathcal{K}$.

Let $\Lambda$ be a subset of $\mathbf{C}$ and assume that every functional Hilbert space on $\Lambda$ is invariant under the map of multiplication by the conjugate of the function $f: \Lambda \rightarrow \mathbf{C}$ defined by $f(z)=z$. The restriction of this map to such'a functional Hilbert space will be denoted by $T_{\bar{z}}$.

Now let $\bar{Q}\left(\Omega, l_{n}^{2}\right)$ denote the set of $l_{n}^{2}$-valued coanalytic functions on the domain $\Omega$ in the plane. Notice that $\bar{Q}\left(\Omega, l_{n}^{2}\right)$ is invariant under multiplication by $\bar{z}$. All functional Hilbert spaces on $\Omega$ under consideration will be invariant under $T_{\bar{z}}$. A functional Hilbert space on $\Omega$ which is a linear subspace of $\bar{Q}\left(\Omega, l_{n}^{2}\right)$ will be called a coanalytic functional Hilbert space on $\Omega$.

A kernel function $K: \Omega \times \Omega \rightarrow \complement\left(l_{n}^{2}\right)$ is called sesquianalytic if $K(\cdot, \cdot)$ is analytic in the first variable and coanalytic in the second variable. Given a sesquianalytic kernel function $K$ on $\Omega$, let $H_{p}(\lambda ; K)$ be the $(p+1) \times(p+1)$-positive operator matrix whose $m, n$-entry is

$$
\frac{\partial^{m}}{m ! \partial z^{m}} \frac{\partial^{n}}{n ! \partial \bar{z}^{n}} K(\lambda, \lambda), \quad 0 \leqslant m, n \leqslant p
$$


We say that the kernel function $K$ is nondegenerate if $H_{p}(\lambda ; K)$ is injective for every $\lambda \in \Omega$ and every positive integer $p$.

Let $K$ be a nondegenerate sesquianalytic kernel function on $\Omega$. Then there exists a coanalytic functional Hilbert space $\mathcal{K}_{K}$ on $\Omega$ such that the nondegenerate kernel function $K$ on $\Omega$ is a reproducing kernel for $\mathcal{K}_{K}$.

A nondegenerate sesquianalytic kernel function $K$ on $\Omega$ is called a generalized Bergman kernel (g.B.k. for brevity) if $T_{\bar{z}} \in \mathcal{L}\left(\mathcal{K}_{K}\right)$ and, for every $\lambda \in \Omega, T_{\bar{z}}^{*}-\lambda$ has closed range and $\operatorname{ran} K(\lambda, \cdot)=\operatorname{ker}\left(T_{z}^{*}-\lambda\right)$. For a detailed treatment of generalized Bergman kernels, the reader is referred to [3]. In particular a g.B.k. $K$ on $\Omega$ is strictly positive [3] and hence $\mathscr{D}(\Omega)$ is dense in $\mathcal{K}_{K}$. The operator $T_{\bar{z}}^{*}$ acting on $\mathcal{K}_{K}$ is called the canonical model associated with $K$. For a proof of the next theorem see [3].

THEOREM 2.1. Let $K$ be a g.B.k. on $\Omega$. If $X$ is in $\left\{T_{\bar{z}}^{*}\right\}^{\prime}$, the commutant of $T_{\bar{z}}^{*}$, then there exists an analytic function $\Phi: \Omega \rightarrow \mathcal{L}\left(l_{n}^{2}\right)$ such that $X K(\lambda, \cdot)=K(\lambda, \cdot) \Phi(\lambda)$, $\lambda \in \Omega$.

3. The weak * closed algebra. Throughout the rest of the paper we let $\Omega$ be a bounded open connected subset of the plane and let $K$ be a g.B.k. on $\Omega$. Let $T=T_{z}^{*} \in \mathcal{L}\left(\mathcal{K}_{K}\right)$ be the canonical model associated with $K$. We further assume that $\sigma(T)=\bar{\Omega}$ is a spectral set for $T$. For convenience we also let $\mathcal{K}=\mathcal{K}_{K}$. The next lemma is the first step towards characterizing the weak * closed algebra generated by $T$.

LEMMA 3.1. Let $\alpha$ be an ordinal number and let $T$ be as before. Then there is an isometry $\Phi_{\alpha}$ from $H^{\infty}\left(\partial \Omega_{\alpha}\right)$ into $\mathcal{L}(\mathcal{H})$.

Proof. Let $h \in H^{\infty}\left(\partial \Omega_{\alpha}\right)$. Since $R\left(\bar{\Omega}_{\alpha}\right)$ is a Dirichlet algebra, there is a sequence $\left\{r_{n}\right\}$ in $R\left(\bar{\Omega}_{\alpha}\right)$ with $r_{n} \rightarrow h \mathrm{w}^{*}$ in $H^{\infty}\left(\partial \Omega_{\alpha}\right)$ and $\sup _{n}\left\|r_{n}\right\|_{\Omega_{\alpha}} \leqslant\|h\|_{\Omega_{\alpha}}$ [5, pp. 5-6]. Because $T$ is von Neumann, $\left\|r_{n}(T)\right\| \leqslant\left\|r_{n}\right\|_{\Omega}$ and since the unit ball of $\mathcal{L}(\mathcal{H})$ is WOT compact, by passing to a subsequence if necessary, we may assume $r_{n}(T) \rightarrow A$ (WOT) for some $A$ in $\mathcal{L}(\mathcal{H})$. Let $f=\sum_{i=1}^{k} K\left(\lambda_{i}, \cdot\right) \xi_{i}$ be in $\mathscr{D}(\Omega)$. Then

$$
r_{n}(T) f=\sum_{i=1}^{k} r_{n}\left(\lambda_{i}\right) K\left(\lambda_{i}, \cdot\right) \xi_{i}
$$

converges weakly to $A f$. Also $\sum_{i=1}^{k} r_{n}\left(\lambda_{i}\right) K\left(\lambda_{i}, \cdot\right) \xi_{i}$ converges in norm to $\sum_{i=1}^{k} h\left(\lambda_{i}\right) K\left(\lambda_{i}, \cdot\right) \xi_{i}$. Define $h(T)=A$. It is easy to see that the definition of $h(T)$ does not depend on the approximating sequence $\left\{r_{n}\right\}$. Now $\left\|r_{n}(T)\right\| \leqslant\|h\|_{\Omega_{\alpha}}$ implies $\|h(T)\| \leqslant\|h\|_{\Omega_{\alpha}}$ Also $h(T) K(\lambda, \cdot) \xi=h(\lambda) K(\lambda, \cdot) \xi, \lambda \in \Omega$. This implies

$$
\|h(\lambda) K(\lambda, \cdot) \xi\| \leqslant\|h(T)\|\|K(\lambda, \cdot) \xi\| \text {. }
$$

Hence $|h(\lambda)| \leqslant\|h(T)\|, \lambda \in \Omega$. So $\|h\|_{\Omega_{\alpha}}=\|h\|_{\Omega} \leqslant\|h(T)\|$. Therefore $\|h(T)\|=$ $\|h\|_{\Omega_{\alpha}}$.

For any operator $S \in \mathcal{L}(\mathcal{H})$ let $\mathbb{Q}_{1}(S)$ denote the WOT sequential closure of $\{p(S): p$ is a polynomial $\}$. We now define inductively for every ordinal number $\alpha$ a set $\mathbb{Q}_{\alpha}(S)$ as follows. If $\mathbb{Q}_{\alpha}(S)$ is defined for some ordinal number $\alpha$, let $\mathbb{Q}_{\alpha+1}(S)$ denote the WOT sequential closure of $Q_{\alpha}(S)$. If $\alpha$ is a limit ordinal and $Q_{\alpha}(S)$ is defined for all $\beta<\alpha$, let $\mathbb{Q}_{\alpha}(S)$ be the WOT sequential closure of $\cup_{\beta<\alpha} \mathbb{Q}_{\beta}(S)$. It is a well-known property of weak * topologies [1] that the spaces $\mathbb{Q}_{\alpha}(S)$ eventually 
become constant; that is, there is a least countable ordinal $\alpha_{0}$ such that $\mathbb{Q}_{\alpha}(S)=$ $\mathbb{Q}_{\alpha_{0}}(S)=\mathbb{Q}(S)$ for $\alpha \geqslant \alpha_{0}$.

Now let $T$ be as before. Applying transfinite induction we will show that for each ordinal number $\alpha$ there exists an isometric isomorphism $\Phi_{\alpha}: H^{\infty}\left(\partial \Omega_{\alpha}\right) \rightarrow Q_{\alpha}(T)$. To see this let $h \in H^{\infty}\left(\partial \Omega_{\alpha}\right)$. Then by Lemma 3.1, there is an operator $h(T)$ in $\mathcal{L}(\mathcal{C})$ such that $\|h(T)\|=\|h\|_{\infty}$. It remains to show that $h(T)$ is actually in $\Theta_{\alpha}(T)$ and $\Phi_{\alpha}$ is onto.

For $\alpha=1$, since $h \in H^{\infty}\left(\Omega_{1}\right)$ there exists a sequence $\left\{p_{n}\right\}$ of polynomials which is uniformly bounded on $\Omega$ and converges to $h$ at each point of $\Omega[6$, p. 521]. Because $T$ is von Neumann, $\left\|p_{n}(T)\right\| \leqslant\left\|p_{n}\right\|_{\Omega} \leqslant M$ for some $M>0$, and since the unit ball of $\mathcal{L}(\mathcal{H})$ is WOT compact, by passing to a subsequence if necessary, we may assume $p_{n}(T) \rightarrow Q\left(\right.$ WOT) for some $Q$ in $\mathcal{L}(\mathcal{H})$. Let $f=\sum_{i=1}^{k} K\left(\lambda_{i}, \cdot\right) \xi_{i}$ be in $\mathscr{D}(\Omega)$. Then

$$
p_{n}(T) f=\sum_{i=1}^{k} p_{n}\left(\lambda_{i}\right) K\left(\lambda_{i}, \cdot\right) \xi_{i}
$$

converges weakly to $Q f$ and in norm to $\sum_{i=1}^{k} h\left(\lambda_{i}\right) K\left(\lambda_{i}, \cdot\right) \xi_{i}$. By definition of $h(T)$ and the fact that $\mathscr{D}(\Omega)$ is dense in $\mathcal{H}$, we conclude that $h(T)=Q \in Q_{1}(T)$.

For a nonlimit ordinal $\alpha$ assume $\Phi_{\alpha-1}: H^{\infty}\left(\partial \Omega_{\alpha-1}\right) \rightarrow \mathbb{Q}_{\alpha-1}(T)$ is an isometry and let $\Phi_{\alpha}: H^{\infty}\left(\partial \Omega_{\alpha}\right) \rightarrow \mathcal{L}(\mathcal{H})$ be as in Lemma 3.1. Let $h \in H^{\infty}\left(\partial \Omega_{\alpha}\right)$ and define $h(T)$ as in Lemma 3.1. We want to show that $h(T) \in \mathbb{Q}_{\alpha}(T)$. Because $h \in H^{\infty}\left(\partial \Omega_{\alpha}\right)$ there is a sequence $\left\{h_{n}\right\}$ in $H^{\infty}\left(\Omega_{\alpha-1}\right)$ which is uniformly bounded on $\Omega$ and converges to $h$ at every point of $\Omega$ [6, Theorem 1, p. 523]. Since $\Phi_{\alpha-1}$ is an isometry, $\left\|h_{n}(T)\right\|=$ $\left\|h_{n}\right\|_{\infty} \leqslant M$ for $M>0$. It is easy to see that $h_{n}(T) \rightarrow h(T)$ (WOT). Therefore $h(T) \in \mathbb{Q}_{\alpha}(T)$.

Suppose $\alpha$ is a limit ordinal and $\Phi_{\alpha}: H^{\infty}\left(\partial \Omega_{\alpha}\right) \rightarrow \mathcal{L}(\mathcal{H})$ is as in Lemma 3.1. Let $h \in H^{\infty}\left(\partial \Omega_{\alpha}\right)$. We will show that $h(T) \in \mathbb{Q}_{\alpha}(T)$. To see this let $\{\beta(n)\}$ be an increasing sequence of ordinals such that $\alpha$ is the least ordinal exceeding every $\beta(n)$. As in the proof of Theorem 2 of Sarason [6, p. 525] there is a sequence $\left\{h_{n}\right\}$ in $H^{\infty}\left(\Omega_{\beta(n)}\right)$ with $h_{n}(\lambda) \rightarrow h(\lambda), \lambda \in \Omega, \sup _{n}\left\|h_{n}\right\|_{\Omega_{\beta(n)}} \leqslant M$ for some $M>0$. Again we can show that $h_{n}(T) \rightarrow h(T)$ (WOT). Since $h_{n}(T) \in \mathbb{Q}_{\beta(n)}(T)$, it follows that $h(T)$ $\in Q_{\alpha}(T)$.

LemMa 3.2. Let $T$ be as before. Then for every ordinal number $\alpha, \Phi_{\alpha}$ is an isometric isomorphism from $H^{\infty}\left(\partial \Omega_{\alpha}\right)$ onto $\mathbb{Q}_{\alpha}(T)$.

Proof. We apply transfinite induction to show that for every ordinal number $\alpha$, $\Phi_{\alpha}$ is onto.

To show that $\Phi_{1}$ is onto, let $R \in Q_{1}(T)$. Then since $R \in\{T\}^{\prime}$, by Theorem 2.1 there exists an analytic function $\Psi: \Omega \rightarrow \mathcal{L}\left(l_{n}^{2}\right)$ such that $R K(\lambda, \cdot)=K(\lambda, \cdot) \Psi(\lambda)$, $\lambda \in \Omega$. By definition of $Q_{1}(T)$ there exists a sequence $\left\{p_{n}\right\}$ of polynomials such that $p_{n}(T) \rightarrow R$ (WOT). Now $\left\|p_{n}(T)\right\| \leqslant M$ for some $M>0$ and $\left\|p_{n}\right\|_{\Omega_{1}}=\left\|p_{n}\right\|_{\Omega}=$ $\left\|p_{n}(T)\right\| \leqslant M$. Also $p_{n}(T) K(\lambda, \cdot) \xi=p_{n}(\lambda) K(\lambda, \cdot) \xi, \lambda \in \Omega$, converges weakly to $R K(\lambda, \cdot) \xi=K(\lambda, \cdot) \Psi(\lambda) \xi$. Therefore

$$
\left\langle p_{n}(\lambda) K(\lambda, \cdot) \xi, K(\lambda, \cdot) \eta\right\rangle=\left\langle p_{n}(\lambda) K(\lambda, \lambda) \xi, \eta\right\rangle
$$


converges to

$$
\langle R K(\lambda, \cdot) \xi, K(\lambda, \cdot) \eta\rangle=\langle K(\lambda, \cdot) \Psi(\lambda) \xi, K(\lambda, \cdot) \eta\rangle=\langle K(\lambda, \lambda) \Psi(\lambda) \xi, \eta\rangle
$$

for every $\xi, \eta \in l_{n}^{2}$. It follows that $p_{n}(\lambda) K(\lambda, \lambda) \rightarrow K(\lambda, \lambda) \Psi(\lambda)$ (WOT). Since $K(\lambda, \lambda)$ is a positive invertible operator [3], it is easy to see that

$$
p_{n}(\lambda) K(\lambda, \lambda)^{-1} K(\lambda, \lambda) \rightarrow K(\lambda, \lambda)^{-1} K(\lambda, \lambda) \Psi(\lambda) \quad \text { (WOT). }
$$

Hence $p_{n}(\lambda) I \rightarrow \Psi(\lambda)$ (WOT). Let $e$ be a fixed unit vector in $l_{n}^{2}$. Then $\left\langle p_{n}(\lambda) e, e\right\rangle \rightarrow$ $\psi(\lambda)$, where $\psi(\lambda)=\langle\Psi(\lambda) e, e\rangle, \lambda \in \Omega$. It is clear that $\psi$ is analytic on $\Omega$. Since $\left\{p_{n}\right\}$ is uniformly bounded on $\Omega$, it follows that $\psi$ is also bounded on $\Omega$, and therefore $\psi \in H^{\infty}(\Omega)$. By Farrell's Theorem [6, p. 521], $\psi$ extends to a bounded analytic function, call it $\psi$ again, on $\Omega_{1}$. It is easy to see that $R=\psi(T)$ and $R K(\lambda, \cdot)=\psi(\lambda) K(\lambda, \cdot), \lambda \in \Omega$.

For a nonlimit ordinal $\alpha$ assume $\Phi_{\alpha-1}$ is onto, let $S \in \mathcal{Q}_{\alpha}(T)$ and choose a sequence $\left\{S_{n}\right\}$ in $\mathbb{Q}_{\alpha-1}(T)$ such that $S_{n} \rightarrow S$ (WOT). By the induction hypothesis, $S_{n} K(\lambda, \cdot)=\psi_{n}(\lambda) K(\lambda, \cdot), \lambda \in \Omega$, where $\psi_{n}: \Omega \rightarrow \mathrm{C}$ is analytic and has an analytic extension to $\Omega_{\alpha-1}$, call it $\psi_{n}$ again. We have $\left\|\psi_{n}\right\|_{\Omega_{\alpha-1}}=\left\|\psi_{n}\right\|_{\Omega}=\left\|S_{n}\right\| \leqslant M$, for some $M>0$. By Theorem 2.1, since $S \in\{T\}^{\prime}, S K(\lambda, \cdot)=K(\lambda, \cdot) \Psi(\lambda), \lambda \in \Omega$, where $\Psi$ : $\Omega \rightarrow \mathcal{L}\left(l_{n}^{2}\right)$ is analytic. It is easy to see that $\psi_{n}(\lambda) I \rightarrow \Psi(\lambda)$ (WOT). Let $e$ be a fixed unit vector in $l_{n}^{2}$. Then $\left\langle\psi_{n}(\lambda) e, e\right\rangle \rightarrow\langle\Psi(\lambda) e, e\rangle, \lambda \in \Omega$. From this we conclude that $\psi_{n}(\lambda) \rightarrow \psi(\lambda)$, where $\psi(\lambda)=\langle\Psi(\lambda) e, e\rangle$ is analytic on $\Omega$. Now $\left\{\psi_{n}\right\}$ forms a normal family in $H^{\infty}\left(\Omega_{\alpha-1}\right)$ and hence there exists a subsequence $\left\{\psi_{n_{h}}\right\}$ that converges uniformly on compact subsets of $\Omega_{\alpha-1}$ to a function $g$ in $H^{\infty}\left(\Omega_{\alpha-1}\right)$. For $\lambda \in \Omega, \psi_{n_{k}}(\lambda) \rightarrow g(\lambda)$. So $g(\lambda)=\psi(\lambda), \lambda \in \Omega$. This proves that $\psi$ has an analytic extension to $\Omega_{\alpha-1}$ and, hence, to $\Omega_{\alpha}$.

Suppose $\alpha$ is a limit ordinal and let $X \in \cup_{\beta<\alpha} \mathbb{Q}_{\beta}(T)$. Then $X \in \mathbb{Q}_{\beta}(T)$ for some $\beta<\alpha$. Also $X K(\lambda, \cdot)=\psi(\lambda) K(\lambda, \cdot), \lambda \in \Omega$, where $\psi$ is a complex-valued analytic function on $\Omega$. From the induction hypothesis it follows that $\psi$ has an analytic extension to $\Omega_{\beta}$ and, since $\Omega_{\alpha} \subset \Omega_{\beta}$, to $\Omega_{\alpha}$.

If there is a sequence $\left\{A_{n}\right\}$ in $\cup_{\beta<\alpha} \mathbb{Q}_{\beta}(T)$ such that $A_{n} \rightarrow A$ (WOT), then $A K(\lambda, \cdot)=K(\lambda, \cdot) \Psi(\lambda), \lambda \in \Omega$, where $\Psi: \Omega \rightarrow \mathcal{L}\left(l_{n}^{2}\right)$ is analytic and $A_{n} K(\lambda, \cdot)=$ $\psi_{n}(\lambda) K(\lambda, \cdot), \lambda \in \Omega$, and $\psi_{n}: \Omega \rightarrow \mathbf{C}$ has an analytic extension to $\Omega_{\alpha}$ by the previous argument. Now $\sup _{n}\left\|A_{n}\right\| \leqslant M$ for some $M>0$, hence $\left\|\psi_{n}\right\|_{\Omega_{\alpha}} \leqslant M$. Also $\psi_{n}(\lambda) I \rightarrow$ $\Psi(\lambda)$ (WOT). Let $e$ be a unit vector in $l_{n}^{2}$. Then $\left\langle\psi_{n}(\lambda) e, e\right\rangle \rightarrow\langle\Psi(\lambda) e, e\rangle$. Let $\psi(\lambda)=\langle\Psi(\lambda) e, e\rangle, \lambda \in \Omega$. Then $\psi_{n}(\lambda) \rightarrow \psi(\lambda), \lambda \in \Omega$, and $\psi$ is analytic on $\Omega$. By Vitali's Theorem $\psi_{n}$ converges on $\Omega_{\alpha}$ to a bounded analytic function $f$, and we have $\psi(\lambda)=f(\lambda), \lambda \in \Omega$. Therefore $\psi$ has an analytic extension to $\Omega_{\alpha}$. Hence $\Phi_{\alpha}$ is onto. Q.E.D.

THEOREM 3.3. Let $T=T_{\bar{z}}^{*}$ be the canonical model associated with a g. B. $k . K$ defined on $\Omega$ such that $\sigma(T)=\bar{\Omega}$ is a spectral set for $T$. Then there is a norm isometric, $w^{*}$-homeomorphic, algebra isomorphism $\Phi$ from $H^{\infty}\left(\partial \Omega_{\gamma}\right)$ onto $\mathbb{Q}(T)$ that takes a polynomial $p$ into $p(T)$. In fact, $\Phi$ is a functional calculus.

Proof. We have already shown that for every ordinal number $\alpha$ there exists an isometric isomorphism $\Phi_{\alpha}: H^{\infty}\left(\partial \Omega_{\alpha}\right) \rightarrow \mathbb{Q}_{\alpha}(T)$. Now let $\alpha_{0}$ be the first uncountable 
ordinal and let $\Phi=\Phi_{\alpha_{0}}: H^{\infty}\left(\partial \Omega_{\alpha_{0}}\right) \rightarrow \mathscr{Q}(T)$. By the Krein-Smulian Theorem [2, p. 26] if we show that $\Phi$ is $\mathrm{w}^{*}$-sequentially continuous, then it is $\mathrm{w}^{*}$-continuous. Let $h_{n}$ and $h$ be in $H^{\infty}\left(\partial \Omega_{\alpha_{0}}\right)$ such that $h_{n} \rightarrow h \mathrm{w}^{*}$. Then $\sup _{n}\left\|h_{n}\right\|_{\Omega_{\alpha_{0}}} \leqslant M$ for some $M>0$ and $h_{n} \rightarrow h$ pointwise on $\Omega_{\alpha_{0}}$. It follows that $h_{n}(T) \rightarrow h(T)$ (WOT) and since $\left\|h_{n}(T)\right\| \leqslant M, h_{n}(T) \rightarrow h(T) \mathrm{w}^{*}$. Since $\Phi$ is an isometry it follows from the KreinSmulian Theorem [2, p. 26] that $\Phi$ is a $w^{*}$-homeomorphism. Since $\Omega_{\alpha_{0}}=\Omega_{\gamma}$ the proof is complete. Q.E.D.

REMARK. What we have done is to find a simply connected domain $\Omega_{0}\left(=\Omega_{\gamma}\right)$ that contains $\Omega$ such that $R\left(\bar{\Omega}_{0}\right)$ is a Dirichlet algebra. As mentioned earlier we can identify $H^{\infty}\left(\partial \Omega_{0}\right)$ and $H^{\infty}\left(\Omega_{0}\right)$. Therefore Theorem 3.3 gives us an isometric isomorphism $H^{\infty}\left(\Omega_{0}\right) \rightarrow \mathscr{Q}(T)$.

The author thanks the referee for his helpful comments.

\section{REFERENCES}

1. S. Banach, Théorie des opérations linéaires, Chelsea, New York, 1955.

2. J. Conway, Subnormal operators, Pitman, London, 1981.

3. R. Curto and N. Salinas, Generalized Bergman kernels and the Cowen-Douglas theory, preprint, 1982.

4. J. Dixmier, Les algèbres d'opérateur dans l'espace hilbertien, Gauthier-Villars, Paris, 1957.

5. D. Sarason, Weak-star density of polynomials, J. Reine Angew. Math. 252 (1972), 1-15.

6. , Weak-star generators of $H^{\infty}$, Pacific J. Math. 17 (1966), 519-528.

Department of Mathematics, University of Calgary, Calgary, Alberta, Canada T2N 1N4 